\title{
Colliding beams of light
}

\author{
B.V.Ivanov日 \\ Institute for Nuclear Research and Nuclear Energy, \\ Tzarigradsko Shausse 72, Sofia 1784, Bulgaria
}

\begin{abstract}
The stationary gravitational field of two identical counter-moving beams of pure radiation is found in full generality. The solution depends on an arbitrary function and a parameter which sets the scale of the energy density. Some of its properties are studied. Previous particular solutions are derived as subcases.
\end{abstract}

04.20.J

\section{INTRODUCTION}

The gravitational field of incoherent light is induced by the energy-momentum tensor $T_{\mu \nu}=\mu_{1} k_{\mu} k_{\nu}$ of pure radiation (null dust) with energy density $\mu_{1}$ and travelling along the null vector $k^{\mu}$. The study of steady beams and pulses of pointed radiation has a long history. In the linear approximation to general relativity this was done about 70 years ago [1.2. Later, Bonnor gave exact solutions for steady and time-dependent beams [3] which belong to the well-known class of pp-waves (algebraically special solutions of Petrov type $\mathrm{N}$ and no expansion) [1]. He showed that beams shining in the same direction do not interact. He also gave solutions with charged null dust [5] and spinning null dust [6]. Counter-moving light beams interact non-linearly, some explanation being proposed in Ref. [7]. Particular exact solutions were found only recently [8,9]. Their Killing vectors necessarily have twist to prevent the focusing of rays. One of these solutions has been also obtained as a limiting case of the double-dust solution [10]. Later, the general double-dust solution was described [11].

In this paper we find the stationary gravitational field of two identical colliding beams of light in full generality. Some of its properties are studied and the previous results are rederived as special cases.

In section 2 the properties of the energy-momentum tensor are studied, the metric and the system of Einstein equations are written down when the energy densities of the beams coincide. Some simplifications are done next and the main equation is obtained. In section 3 a new radial variable is introduced and two methods for the derivation of the general solution are given. The explicit formulas depend on an arbitrary function and a free parameter. The consequences of elementary flatness are studied. In section 4 an algorithm for simple algebraic solutions is presented and the Kramer solutions are derived as an example. The difficulties in the second method, which contains the important case of constant energy density, are clarified. In section 5 some of the properties of the general and the particular solutions are studied. The expansion, the acceleration and the vorticity of $k^{\mu}$ are given in the most general metric. A criterion for the appearance of closed timelike curves (CTC) is given. The static solution of Kramer does not have CTC but its stationary counterpart does have them. Section 6 contains some discussion. A comparison is made between the general double-dust solution and the general solution for colliding light.

\section{ENERGY-MOMENTUM TENSOR AND FIELD EQUATIONS}

We shall work in the stationary formulation of the problem when the cylindrically symmetric metric is given by 4,8

$$
d s^{2}=-e^{2 u}(d t+A d \varphi)^{2}+e^{-2 u}\left[e^{2 k}\left(d x^{2}+d z^{2}\right)+W^{2} d \varphi^{2}\right]
$$

and there is only radial $x$-dependence. The static metric may be obtained by complex substitution; $t \rightarrow i z, z \rightarrow$ $i t, A \rightarrow i A$. The energy-momentum tensor of the two beams reads

$$
\begin{aligned}
& T_{\mu \nu}=\mu_{1} k_{\mu} k_{\nu}+\mu_{2} l_{\mu} l_{\nu}, \\
& k^{\mu} k_{\mu}=0, \quad l^{\mu} l_{\mu}=0,
\end{aligned}
$$

*E-mail: boyko@inrne.bas.bg 


$$
k^{\mu} l_{\mu}=-1 .
$$

Here $\mu_{i}$ are the energy density profiles of the beams and $k^{\mu}, l^{\mu}$ are their 4 -velocities, which are null and normalized as in Eq. (4). As will be shown later, there is only one solution with the minimal set of two non-zero components. On the other hand, naturally $k^{x}=l^{x}=0$. Therefore, let us introduce

$$
k^{t}=l^{t}=V, \quad k^{\varphi}=l^{\varphi}=\Phi, \quad k^{z}=-l^{z}=Z
$$

and $T=V+A \Phi$. The beams move in the positive or negative $z$-direction. For stationary metrics they should have the same twist $\Phi$, otherwise the two conditions in Eq. (3) lead to contradiction. In the static picture the twists are opposite. The obvious relation $T_{\mu}^{\mu}=0$ and Eq. (4) give

$$
\begin{gathered}
e^{2 u} T^{2}-e^{-2 u} W^{2} \Phi^{2}=e^{2(k-u)} Z^{2}, \\
e^{2 u} T^{2}-e^{-2 u} W^{2} \Phi^{2}+e^{2(k-u)} Z^{2}=1 .
\end{gathered}
$$

Their combinations express $Z$ and $\Phi$ in terms of the other quantities

$$
\begin{gathered}
Z=\frac{1}{\sqrt{2}} e^{u-k}, \\
W^{2} \Phi^{2}=e^{2 u}\left(e^{2 u} T^{2}-\frac{1}{2}\right) .
\end{gathered}
$$

The covariant conservation of the energy-momentum tensor (which follows from the field equations) reads

$$
T_{\nu ; \mu}^{\mu}=\frac{1}{\sqrt{-g}} \frac{\partial\left(T_{\nu}^{\mu} \sqrt{-g}\right)}{\partial x^{\mu}}-\frac{1}{2}\left(g_{\mu \lambda}\right)_{\nu} T^{\mu \lambda}=0
$$

where $g$ is the metric's determinant. In our case $T_{\nu}^{r}=0$ and the first term vanishes. The second yields

$$
\left(e^{2 u}\right)^{\prime} V^{2}+2\left(A e^{2 u}\right)^{\prime} V \Phi-\left(e^{-2 u} W^{2}-e^{2 u} A^{2}\right)^{\prime} \Phi^{2}=2(k-u)^{\prime} e^{2(k-u)} Z^{2},
$$

where' means $x$-derivative. The restriction $\Phi=0$ immediately gives the particular solution $k=2 u$, as announced above.

To write the Einstein equations we use the combinations of Ricci tensor components utilized in Refs. 11, 12, 13, 14,. We shall work, however, with curved mixed components $R_{\nu}^{\mu}$. The only non-trivial ones are the diagonal components and $R_{\varphi}^{t}, R_{t}^{\varphi}$. The Einstein equations for the corresponding $T_{\nu}^{\mu}$ components give after some rearrangement

$$
\begin{gathered}
\frac{W^{\prime \prime}}{W}=8 \pi \rho e^{2(k-u)}, \\
2 \frac{k^{\prime} W^{\prime}}{W}-2 u^{\prime 2}+\frac{e^{4 u} A^{\prime 2}}{2 W^{2}}=0, \\
u^{\prime \prime}+\frac{u^{\prime} W^{\prime}}{W}+\frac{e^{4 u} A^{\prime 2}}{2 W^{2}}=16 \pi \rho e^{2 k} T^{2}, \\
k^{\prime \prime}+u^{\prime 2}+\frac{e^{4 u} A^{\prime 2}}{4 W^{2}}=16 \pi \rho e^{2 k-4 u} W^{2} \Phi^{2}, \\
\left(\frac{e^{4 u} A^{\prime}}{W}\right)^{\prime}=-32 \pi \rho e^{2 k} W \Phi T .
\end{gathered}
$$

Here $2 \rho=\mu_{1}+\mu_{2}$. Units are used with $G=c=1$. The equations for the rest $T_{\nu}^{\mu}$ components are satisfied either identically or when $\left(\mu_{1}-\mu_{2}\right) Z=0$. Therefore, we accept in the following that $\mu_{1}=\mu_{2}=\rho$. There are 7 equations (8,9,12-16) for 8 unknowns $u, k, W, A, \rho, V, \Phi, Z$. Eq. (13) gives an expression for $A^{\prime}$ 


$$
A^{\prime}= \pm 2 e^{-2 u} W \sqrt{B}, \quad B=u^{\prime 2}-\frac{k^{\prime} W^{\prime}}{W},
$$

which allows to eliminate $A^{\prime}$ from Eqs. (14-16). The difference between Eqs. $(14,15)$ leads to a relation between $k-u$ and $W$

$$
k^{\prime}=u^{\prime}+\frac{1-W^{\prime}}{W}, \quad e^{k-u}=\frac{e^{\int \frac{d x}{W}}}{W} .
$$

An unimportant constant has been set to 1 in the first of these equations by rescaling the $x$-coordinate. Now Eqs. $(12,18)$ show that $\rho$ can be expressed through $W$

$$
8 \pi \rho=W W^{\prime \prime} e^{-2 \int \frac{d x}{W}}
$$

or $W$ can be found as a solution of a rather complicated equation if $\rho$ was given

$$
\left(W W^{\prime \prime}\right)^{\prime}-2 W^{\prime \prime}-(\ln \rho)^{\prime} W W^{\prime \prime}=0 .
$$

Eq. (8) defines $Z$ in terms of $W$, Eqs. (14,9) express $T$ and $\Phi$ in terms of $u$ and $W$. Thus all characteristics of the problem have expressions as functions of $W$ or $u$ and $W$. The arbitrary function, however, can be only one and a relation between them follows from the crucial Eq. (16). While Eqs. $(14,15)$ are not sensitive to the sign of $A^{\prime}$ in Eq.(17), Eq.(16) should be solved for both signs of $A^{\prime}$, leading to different solutions. They are encompassed by taking the squares of both sides of Eq. (16) and inserting $T^{2}, \Phi^{2}$ and $A^{\prime}$ from Eqs. $(14,15,17)$ to obtain

$$
\left(4 u^{\prime} B+B^{\prime}\right)^{2}=4 B\left(u^{\prime \prime}+\frac{u^{\prime} W^{\prime}}{W}+2 B\right)\left(u^{\prime \prime}+\frac{u^{\prime} W^{\prime}}{W}+2 B-\frac{W^{\prime \prime}}{W}\right) .
$$

This equation involves only $u$ and $W$ due to Eqs. $(17,18)$. From the definition of $T$ it follows that if $A, \Phi, T$ is a solution of Eq. (16), so is $-A,-\Phi, T$. In other words, the equations are invariant to a change in the direction of dragging and the sign of the twist of the pure radiation's 4-velocity.. This is different from the vacuum case 15 and the case of perfect fluid [13], where the r.h.s. of Eq. (16) vanishes and the direction of rotation is not significant. In our case the two signs of $A$ (or $\Phi$ ) lead to a double valuedness of the energy density $\rho$, as will be seen from Eqs. (29, 37) below.

\section{THE GENERAL SOLUTION}

Let us introduce the new radial variable $p=W^{\prime}$ and denote the $p$-derivatives with a subscript. Eq. (12) shows that this is possible when $\rho \neq 0$. Let us introduce also the important function $X$

$$
X=W W^{\prime \prime}=\frac{p W}{W_{p}} .
$$

Another fundamental quantity is $f$, introduced by

$$
u^{\prime}=\frac{f}{W}
$$

Then $B$ becomes

$$
B=\frac{b}{W^{2}}, \quad b=f^{2}-p f+p^{2}-p,
$$

where $b>0$ in order to have a real $A^{\prime}$. This constrains to some extent the arbitrary function $f$. After the use of the last three equations the main Eq. (21) turns into a quadratic equation for $X$

$$
\begin{aligned}
& C_{1} X^{2}+C_{2} X+C_{3}=0, \\
& C_{1}=b_{p}^{2}-4 b f_{p}\left(f_{p}-1\right),
\end{aligned}
$$




$$
\begin{gathered}
C_{2}=4 b\left[(2 f-p) b_{p}-2 b\left(2 f_{p}-1\right)\right], \\
C_{3}=4 b^{2}\left[(2 f-p)^{2}-4 b\right] .
\end{gathered}
$$

Every function $f$ corresponds to two solutions

$$
X_{a, b}=\frac{-C_{2} \pm \sqrt{D}}{2 C_{1}}, \quad D=C_{2}^{2}-4 C_{1} C_{3} .
$$

The coefficients $C_{i}$ are simplified when the definition of $b$ is used

$$
\begin{gathered}
C_{1}=p(4-3 p) f_{p}^{2}+2(3 p f-2 f-p) f_{p}+(2 p-1-f)^{2}, \\
C_{2}=4 b\left[p(4-3 p) f_{p}+3 p f-2 f-p\right] \\
C_{3}=4 b^{2} p(4-3 p) .
\end{gathered}
$$

It is interesting that only 4 combinations of $p$ and $f$ enter these expressions. Another surprising fact is that $D$ factors out into terms that do not contain $f_{p}$

$$
D=16 b^{2}\left[(3 p f-2 f-p)^{2}-p(4-3 p)(2 p-1-f)^{2}\right]=64 b^{3}(p-1)(3 p-1) .
$$

$X$ must be real, hence $D \geq 0$. This restricts the $p$ coordinate outside the interval $(1 / 3,1)$. The position of the axis is at $p=1$, because $W(x) \rightarrow x$ when $x \rightarrow 0$. Thus the realistic interval is given by $p \geq 1$. It is also obvious that Eq. $(25)$ is quadratic with respect to $f_{p}$

$$
p(4-3 p) X^{2} f_{p}^{2}+\left[2(3 p f-2 f-p) X^{2}+4 p(4-3 p) b X\right] f_{p}+(2 p-1-f)^{2} X^{2}
$$

$+4(3 p f-2 f-p) b X+4 p(4-3 p) b^{2}=0$.

After these remarks we are ready to present the general solution of the problem of two colliding light beams. There are two methods to do this. The easier one is to choose an arbitrary function $f(p)$ and find the two $X$ from the root formula (29). Then Eqs. (23,18,22,17,19,8,9 and 14) yield respectively $u, k, W, A, \rho, Z, \Phi$ and $V$

$$
\begin{gathered}
u=\int \frac{f}{X} d p, \quad k=\int \frac{f+1-p}{X} d p+k_{0}, \\
W=W_{0} e^{\int \frac{p}{X} d p}, \quad A=2 \int_{1}^{p} \frac{\sqrt{b}}{X} W e^{-2 u} d p, \\
\rho=\rho_{0} X e^{-2 \int \frac{d p}{X}-2 k_{0}}, \quad Z=\frac{1}{\sqrt{2}} e^{\int \frac{p-1}{X} d p-k_{0}}, \\
T^{2}=\frac{2 b+f_{p} X}{2 X} e^{-2 u}, \\
\Phi^{2}=\frac{2 b+\left(f_{p}-1\right) X}{2 W_{0}^{2} X} e^{2 u-2 \int \frac{p}{X} d p}, \quad V=T-A \Phi .
\end{gathered}
$$

The radial metric component $g_{x x}$ becomes $g_{p p}$

$$
g_{p p}=e^{2(k-u)} \frac{W^{2}}{X^{2}} .
$$

One can further pass from $p$ to the physical length $L$, so that $g_{L L}=1$ 


$$
L=\int \sqrt{g_{p p}} d p=W_{0} e^{\int \frac{d p}{X}} .
$$

The constant $W_{0}$ is positive by definition. The limits of the integral for $A$ and the integration constant $k_{0}$ are determined by the elementary flatness condition

$$
\lim _{x \rightarrow 0} \frac{e^{u-k}}{x}\left(e^{-2 u} W^{2}-e^{2 u} A^{2}\right)^{1 / 2}=1 .
$$

$W$ and $A$ should vanish at the axis. One can see from Eq. (17) that $A=o(W)$ when $x \rightarrow 0$ even if there is a pole in $B$. Therefore, it can be neglected in Eq. (42) like in many other problems 11, 13, 14]. The function $f$ (and $b$ ) also should vanish at the axis to keep $u^{\prime}$ regular. Using the l'Hôpital rule and passing to $p$, Eq. (42) becomes $(2 p-f-1) e^{-k} \rightarrow 1$ when $p \rightarrow 1$. This gives $k(1)=0$ which fixes $k_{0}$. Inserting $W$ and $\rho$ from Eqs. $(36,37)$ into Eq. (12) supplies the relation

$$
8 \pi \rho_{0} W_{0}^{2}=1 .
$$

It indicates that $\rho_{0}$ is positive. Eq. (43) has been used in Eqs. $(38,39)$. Eqs. $(37,43)$ show that $X>0$, which places another restriction on $f$. Furthermore, the r.h.s. of Eqs. $(38,39)$ should be positive, leading to the inequality $2 b+\left(f_{p}-1\right) X \geq 0$. Finally, we have kept $A$ positive in Eq. (36), allowing for a change in the sign of $\Phi$ instead. It is determined by the sign of the l.h.s. of Eq. (16), equivalent to the sign of $(4 f-2 p) b+b_{p} X$.

The second, more natural but more difficult method, is to provide the sum of the density profiles $2 \rho$. Then from Eq. (20) or (37) it follows that $X$ satisfies a linear first-order differential equation

$$
X_{p}-(\ln \rho)_{p} X-2=0 .
$$

Its boundary condition states that $X(1)=0$, due to $W(1)=0$. Its solution is

$$
X=2 \rho \int_{1}^{p} \frac{d p}{\rho} .
$$

The next step is to determine $f$ from Eq. (34). This is, in fact, a first-order, highly non-linear equation, whose solutions seem to exist only in numerical form. Its boundary condition is $f(1)=0$. After $X$ and $f$ are found, the rest of the task is fulfilled by Eqs. (35-40) where the two methods coincide.

The general solution thus depends on one arbitrary function $f$ (satisfying several positivity requirements) or a positive $\rho$ and the free parameter $\rho_{0}$. The situation is similar to the general double-dust solution [11. The constant $\rho_{0}$ sets the scale of the energy density. The form of the solution is determined by Eq. (25) or its equivalent Eq. (34). They do not contain $\rho_{0}, W_{0}$ or any other parameters. An explicit solution needs the explicit calculation of only three integrals, $\int \frac{d p}{X}, \int \frac{p d p}{X}$ and $\int \frac{f d p}{X}$.

\section{PARTICULAR SOLUTIONS}

Let us search for some simple concrete solutions. Following the first method, let us choose $f$ in such a way that $D$ becomes a square leading to an algebraic $X$. According to Eq. (33) we must have

$$
b=(p-1)(3 p-1) b_{1}^{2},
$$

where $b_{1}$ is a polynomial. Eq. (24) shows that the simplest possibility is constant $b_{1}$ and linear $f$. There are four such cases

$$
\begin{gathered}
f_{1}=1-p, \quad k=2 u, \\
f_{2}=\frac{p-1}{2}, \quad k=-u, \\
f_{3}=\frac{p+1}{2}, \quad k=3 u-2 \ln W,
\end{gathered}
$$




$$
f_{4}=2 p-1, \quad k=\ln W .
$$

Then Eq. (29) yields 8 solutions for $X$

$$
\begin{gathered}
X_{1 a}=(p-1)(3 p-1), \quad X_{1 b}=\frac{p(p-1)(3 p-1)(4-3 p)}{3 p^{2}-4 p+2}, \\
X_{2 a}=\frac{(p-1)(3 p-1)(4-3 p)}{6 p-5}, \quad X_{2 b}=\frac{p(p-1)(3 p-1)}{2 p-1}, \\
X_{3 a}=-X_{2 b}, \quad X_{3 b}=-X_{2 a}, \\
X_{4 a}=-X_{1 b}, \quad X_{4 b}=-X_{1 a} .
\end{gathered}
$$

The first four coincide with solutions I-IV from Ref. 91. As was mentioned already, realistic solutions have positive $X$, at least in some region around the axis. Setting $p=R^{2}+1$ we see that $X_{1 a}$ and $X_{2 b}$ are positive everywhere, while $X_{1 b}$ and $X_{2 a}$ are positive for $1<p<4 / 3$. These solutions are suitable for interior solutions. The other four solutions are physically unrealistic. $X_{4 b}$ and $X_{3 a}$ are negative everywhere, while $X_{4 a}$ and $X_{3 b}$ are negative on the axis and in its vicinity. This fact was noticed in Ref. [9].

We have shown that when $\Phi=0, k=2 u$. The opposite is not true since $X_{1 b}$ leads to non-vanishing $\Phi$. Thus the solution with $f_{1}$ and $X_{1}$ is the simplest possible solution. It was thoroughly investigated 8.96,10]. It is worth to point out how it arises from the general formalism. When $k=2 u, u$ also picks up an integration constant $k_{0} / 2$, while the one in $\rho$ becomes $-k_{0}$. Eqs. (35-40) give

$$
\begin{gathered}
e^{2 u}=Y^{-2 / 3}, \quad e^{2 k}=Y^{-4 / 3}, \quad e^{k_{0}}=2^{2 / 3}, \\
W^{2}=W_{0}^{2} R^{2} Y^{-1 / 3}, \quad A=2^{1 / 3} W_{0} R^{2}, \\
\rho=2^{4 / 3} \rho_{0} Y^{2}, \quad \Phi=0, \\
Z=V=T=\frac{1}{\sqrt{2}} Y^{1 / 3},
\end{gathered}
$$

where

$$
Y=\frac{1}{2}(3 p-1)=1+\frac{3}{2} R^{2} .
$$

These results coincide with the results of Refs. [8.96] when the identifications

$$
8 \pi \rho_{0}=\frac{2^{2 / 3}}{3} \lambda^{2}, \quad R=\sqrt{\frac{2}{3}} \lambda r
$$

are made. The free parameter $\lambda$ reflects the free scale of the energy density.

Eq. (46) sets a combinatorial problem from polynomial algebra, which probably has additional solutions with more complex $f$. Furthermore, when $f$ and $X$ are given analytically, the integrals in the metric may be calculated numerically and a solution, partially explicit, partially numerical, obtained.

The second method for generating solutions also has an analytical start by choosing $\rho$. Then $X$ is found comparatively easy from Eq. (45). Here are four examples

$$
\begin{gathered}
\rho_{1}=\text { const }, \quad X_{1}=2(p-1), \\
\rho_{2}=p^{-\alpha}, \quad X_{2}=\frac{2}{\alpha+1}\left(p-p^{-\alpha}\right), \\
\rho_{3}=c-p, \quad X_{3}=2(c-p) \ln \frac{c-1}{c-p}, \\
\rho_{4}=e^{-p}, \quad X_{4}=2\left(1-e^{1-p}\right) .
\end{gathered}
$$

The constants fulfil $c>1, \alpha>0$. The first case is the simplest from a physical point of view - collision of beams of constant energy density up to some $p_{0}$, where a matching to the vacuum stationary solution should be done. Unfortunately, even for such simple $X$ Eq. (34) is so complicated that we have been unable to solve it even numerically. This hopefully can be done on a more powerful computer platform. 


\section{SOME PROPERTIES OF THE GENERAL SOLUTION}

We have calculated with the help of GRTensor the characteristics of the vector field $k^{\mu}$ in the general stationary cylindrically symmetric metric (1). The shear's expression is too lengthy to be displayed. The expansion $\Theta$, the acceleration $a^{\mu}$ and the vorticity vector $w^{\mu}$ read

$$
\begin{gathered}
\Theta=W^{-1}\left[(W \Phi)^{\prime}-2 W \Phi\left(u^{\prime}-k^{\prime}\right)\right], \\
a^{t}=\Phi\left(V^{\prime}+2 u^{\prime} V+\frac{e^{4 u} V A A^{\prime}}{W^{2}}\right), \\
a^{\varphi}=-\frac{e^{4 u} V \Phi A^{\prime}}{W^{2}}, \\
a^{x}=u^{\prime} e^{4 u-2 k} V^{2}+\left(u^{\prime}-k^{\prime}\right)\left(Z^{2}-\Phi^{2}\right)+\Phi \Phi^{\prime}, \\
w^{t}=\frac{e^{2 u}}{2 W}\left[2\left(2 u^{\prime}-k^{\prime}\right) A V Z+Z A V^{\prime}+Z A^{\prime} V-Z^{\prime} A V\right], \\
w^{\varphi}=-\frac{e^{2 u}}{2 W}\left[2\left(2 u^{\prime}-k^{\prime}\right) V Z+Z^{\prime}\right], \\
w^{x}=0, \\
w^{z}=\frac{e^{6 u-2 k} A^{\prime} V^{2}}{2 W} .
\end{gathered}
$$

When $\Phi=0$ these formulas coincide with those from Ref. [11]. Both $\Theta$ and $a^{\mu}$ are proportional to $\Phi$ and vanish in this case except for $a^{x}$, which becomes

$$
a^{x}=u^{\prime} e^{4 u-2 k} V^{2}+\left(u^{\prime}-k^{\prime}\right) Z^{2} .
$$

It also vanishes for the general double-dust solution and for the only case $k=2 u$ of colliding light with $\Phi=0$. The motion of the source is therefore geodesic. When $\Phi \neq 0$ Eq. (8) shows that

$$
a^{z}=\left(k^{\prime}-u^{\prime}\right) \Phi Z,
$$

which vanishes only in the vacuum case $k=u$. The expansion does not vanish automatically when the general solution is inserted in Eq. (65). Vorticity does not depend on $\Phi$ at all and Eqs. (70-73) coincide with the first relations in Eqs. (28-30) from Ref. [11].

It is well known that CTC exist when $g_{\varphi \varphi}<0$. We have

$$
g_{\varphi \varphi}=e^{2 u}\left(e^{-4 u} W^{2}-A^{2}\right)
$$

and the general solution possesses CTC when

$$
e^{-2 u} W<\int_{1}^{p} \frac{2 \sqrt{b}}{X} e^{-2 u} W d p
$$

For the concrete solution (55-60) this yields 


$$
r^{2}\left(1+\lambda^{2} r^{2}\right)^{-2 / 3}\left(1-\frac{1}{3} \lambda^{2} r^{2}\right)<0,
$$

which is satisfied when $r>\sqrt{3} / \lambda$, so that there are CTC. In the static case $Y=1-\lambda^{2} r^{2}$ and inequality (78) becomes

$$
r^{2} Y^{-2 / 3}\left(1+\frac{1}{3} \lambda^{2} r^{2}\right)<0 .
$$

The l.h.s. is always positive and consequently there are no CTC. This is also true for the exterior solution - the Levi Civita metric. In the stationary case this role is played by the Lewis solution 15. It possesses three distinct classes and only the Weyl class is locally, but not globally static. It seems possible to induce the Lewis class of the exterior solution when $\rho_{0}$ is big enough. This class contains CTC. This discussion of exterior solutions is valid also for the general solution. However, the vacuum solution is not a subcase of the general solution. It has $\rho=0, W=x$, so that $p$ is not a variable but a constant.

\section{DISCUSSION}

We have obtained in this paper the general cylindrically symmetric and stationary (no time dependence) metric when $T_{\mu \nu}$ is given by Eqs. (2-4) and $\mu_{1}=\mu_{2}$. The components of the null vectors and the energy density are found simultaneously with the metric. Although the solution looks quite complicated, minimal assumptions have been made along its derivation. Diagonal metric does not allow colliding solutions, hence, the reason for the appearance of $A$. It prevents the focusing of rays and the appearance of singularity [8,9]. In the case of dust two non-zero components of $k^{\mu}$ are enough for a general solution with arbitrary energy density [1]. In the case of light they give a solution with specific density profile and a third component $\Phi$ is necessary. In the dust case the field equations decouple and can be solved one by one, giving, however, an involved expression for the energy density in terms of the arbitrary function. In the light case there is an additional quadratic non-linearity, because the square of Eq. (16) must be taken in order to encompass all solutions. It reflects further in the quadratic algebraic Eq. (25) for $X$ or the quadratic differential Eq. (34) for $f_{p}$. One can choose arbitrary positive $\rho$ and find $X$ easily. The expression for $f$ and for those parts of the metric depending on it, however, can be only numerical. The other option is to choose $f$, but then there are several inequalities to be satisfied for a realistic solution.

An important property is the traditional appearance of CTC in rotating cylindrically symmetric solutions, which forms one of the differences between the stationary and the general static solution. This takes place both in the interior and the exterior.

In conclusion, this paper can be considered as a further step in the study of the collision of the gravitational fields of beams of coherent or incoherent light. The award motivating the pursuit of this issue is the existence of singularity-free colliding solutions [8].

[1] Tolman R C, Ehrenfest P and Podolsky B 1931 Phys. Rev. 37602

[2] Tolman R C 1934 Relativity, Thermodynamics and Cosmology (Oxford: Clarendon Press)

[3] Bonnor W B 1969 Comm. Math. Phys. 13163

[4] Kramer D, Stephani H, Herlt E and MacCallum M A H 1980 Exact Solutions of Einstein's Field Equations (Cambridge: Cambridge University Press)

[5] Bonnor W B 1970 Int. J. Theor. Phys. 357

[6] Bonnor W B 1970 Int. J. Theor. Phys. 3257

[7] Faraoni V and Dumse R M 1999 Gen. Rel. Grav. 3191

[8] Kramer D 1998 Class. Quantum Grav. 15 L73

[9] von der Gönna U and Kramer D 1999 Gen. Rel. Grav. 31349

[10] Kramer D 2002 Class. Quantum Grav. 192257

[11] Ivanov B V 2002 gr-qc/0209032

[12] Sklavenites D 1999 Class. Quantum Grav. 162753

[13] Ivanov B V 2002 Class. Quantum Grav. 193851

[14] Ivanov B V 2002 Class. Quantum Grav. 195131

[15] Lewis T 1932 Proc. R. Soc. A 136176 